\title{
Acidente Vascular Cerebral e sequelas fonoaudiológicas: atuaçáo em área hospitalar
}

\author{
Stroke followed by speech and language sequels: Hospital Procedures
}

Aline Jacques ${ }^{1}$, Maria Cristina de Almeida Freitas Cardoso ${ }^{2}$

\section{RESUMO}

Objetivo. Realizar um levantamento das possíveis seqüelas fonoaudiológicas dos pacientes internados por Acidente Vascular Cerebral nas enfermarias do Hospital Parque Belém, destinando-se diretamente à ampliaçáo do conhecimento dos profissionais de saúde pública a respeito de tal tema e auxiliando-os, assim, em futuros encaminhamentos e atendimentos a esta populaçáo, dada a importância do profissional da fonoaudiologia frente a estas seqüelas. Método. Estudo transversal, observacional, individual, não comparado e contemporâneo, avaliando a prevalência de seqüelas fonoaudiológicas (disfagias e distúrbios de linguagem) em pacientes acometidos por Acidente Vascular Cerebral, internados no Hospital Parque Belém em Porto Alegre, no período de março a maio de 2007. Resultados. Foram avaliados 26 (100\%) pacientes com Acidente Vascular Cerebral (88,5\% isquêmicos e 11,5\% hemorrágicos), encontrando-se a prevalência de $42,3 \%$ com disfagia e $30,8 \%$ com distúrbio de linguagem. Foi analisada ainda, a variação de saturaçáo destes pacientes, verificando-se incidência de dessaturação em $57,7 \%$ do total de pacientes. Conclusáo. Pacientes acometidos por AVC apresentam alta prevalência de problemas de deglutição e linguagem, indicando a relevância da atuaçáo do fonoaudiólogo em pelo menos duas de suas áreas de intervenção (motricidade orofacial - disfagias; distúrbios de linguagem).

Unitermos. Transtornos de deglutição, Linguagem, Fonoaudiologia.

Citaçáo. Jacques A, Cardoso MCAF. Acidente Vascular Cerebral e sequelas fonoaudiológicas: atuação em área hospitalar.

\begin{abstract}
Objective. To survey the possible speech and language sequelae of patients hospitalized for cerebrovascular accidents in the wards of the Parque Belém Hospital, targeted at increasing the knowledge of public health professionals about this issue and, thus, providing assistance in future referrals and treatments for this group, given the importance of the speech-language pathology professional facing these sequelae. Method. A cross-sectional, observational, individual, not comparing and contemporary, assessing the prevalence of speech and language sequelae (dysphagia and language disorders) in patients affected by cerebrovascular accidents, hospitalized at the Parque Belém Hospital in Porto Alegre, from March to May 2007. Results. A total of 26 (100\%) patients with cerebrovascular accidents (88.5\% ischemic and $11.5 \%$ bleeding) were evaluated, and the prevalence of dysphagia in $42.3 \%$ and $30.8 \%$ with language impairment was found. The variation of the saturation of these patients was also analyzed, and an incidence of desaturation in $57.7 \%$ of the patients was seen. Conclusion. Stroke patients present high prevalence of dysphagia and language impairments, that leads to the relevance of the speech-language pathology professional in at least two of its areas of intervention (orofacial motricity - dysphagia; language impairments).
\end{abstract}

Keywords. Deglutition Disorders, Language, Speech, Language and Hearing Sciences.

Citation. Jacques A, Cardoso MCAF. Stroke followed by speech and language sequels: Hospital Procedures.
Trabalho realizado no Hospital Parque Belém, Porto Alegre-RS, Brasil. 1. Fonoaudióloga, mestranda em Distúrbios da Comunicação Humana pela UFSM, Santa Maria-RS, Brasil.

2. Fonoaudióloga, docente do curso de Fonoaudiologia no Centro Universitário Metodista - IPA, doutora em Gerontologia Biomédica pela PUCRS, Porto Alegre-RS, Brasil.
Endereço para correspondência: Aline Jacques

R. Coronel Niederauer, 747/408 CEP 97500-600, Santa Maria-RS, Brasil. Tel.: (55) 9966-5700

E-mail: alinejacques@yahoo.com.br 


\section{INTRODUÇÃO}

O Acidente Vascular Cerebral (AVC) é um déficit neurológico (transitório ou definitivo) em uma área cerebral secundária à lesão vascular. Trata-se de uma doença circulatória nas artérias cerebrais, que possui etiologia diversa, cujos fatores que contribuem para a sua ocorrência são: genéticos, clínicos e ambientais ${ }^{1-5}$.

$\mathrm{O}$ AVC pode ser classificado em isquêmico, quando o sangue é impossibilitado de passar para determinada área do cérebro em função de uma redução do fluxo sanguíneo ou por uma obstrução no vaso, e hemorrágico, quando ocorre um extravasamento sanguíneo ${ }^{2,3}$.

Esta doença é a principal causa de morte no Brasil, quanto a maior causadora de deficiência motora adquirida, sendo responsável por até $80 \%$ das incapacidades, e por trazer, ainda, outras alteraçóes como os distúrbios de fala ou de linguagem e distúrbios de deglutição ${ }^{6,7}$.

Os distúrbios de linguagem encontrados são denominados como afasia, que pode ser conceituada como a perda completa ou parcial da condição de expressar-se através da fala, da escrita ou de gestos, e interferindo até a capacidade de compreensão oral e escrita ${ }^{8}$.

A Afasia está englobada em uma desordem neurofisiológica, envolvendo os mecanismos cerebrais, que pode vir acompanhada de alteraçóes comportamentais, intelectuais e emocionais, que refletem nas atitudes e na personalidade do indivíduo ${ }^{9,10}$.

Os distúrbios da fala, classificados em problemas de fonação, articulação, ressonância e/ou prosódia, quando originados de uma lesão neurológica são divididos em apraxia ou dispraxia oral e disartria. As apraxias orais acontecem por um déficit nas habilidades de sequencialização dos movimentos das estruturas orofaciais dificultando, dessa forma, o posicionamento correto das estruturas para produção voluntária da fala ${ }^{11,12}$.

A disartria ocorre através de uma lesão no sistema nervoso central e/ou periférico, ocasionando alteração de cinco bases motoras: respiração, fonaçâao, ressonância, articulação e prosódia, debilitando, portanto, a comunicação oral em função dos distúrbios no controle muscular dos mecanismos envolvidos na fala ${ }^{12}$.
Os distúrbios de deglutição são conceituados como uma alteração no processo de deglutição, interferindo no transporte do bolo alimentar da boca até o estômago e classificados em orofaríngeas e esofageanas ${ }^{13}$.

As disfagias neurogênicas ocorrem com muita frequência no AVC. Estas apresentam maiores alteraçôes nas fases oral e faríngea da deglutição, e estão relacionadas a um alto grau de morbidade e mortalidade, pois frequentemente causam alteraçóes nutricionais e aspiração traqueobrônquica de saliva, secreçóes ou alimentos, levando a complicaçôes clínicas de hidratação, desnutrição e risco de pneumonias aspirativas e de repetiçăo ${ }^{14,15}$.

Através da análise da avaliação clínica e instrumental o fonoaudiólogo irá focar seus procedimentos terapêuticos, entre os mais adequados, facilitando, desta maneira, a ocorrência de mecanismos de plasticidade, na reabilitação do paciente que sofreu lesão cortical decorrente de AVC.

Pesquisas apontam que a plasticidade é um atributo do tecido nervoso que se estende no decorrer da vida. Portanto, destaca-se a importância de o profissional que atua na reabilitação ter conhecimento sobre mapeamento entre a atividade do cérebro, o comportamento motor e os mecanismos de plasticidade ${ }^{16}$.

A avaliação e o gerenciamento da equipe fonoaudiológica concomitante ao trabalho multidisciplinar, são de fundamental importância para os pacientes acometidos por $\mathrm{AVC}^{17}$.

Este estudo trata sobre o AVC e as possíveis sequelas fonoaudiológicas de pacientes, internados no Hospital Parque Belém (HPB) de Porto Alegre (RS), hospital geral cuja maior demanda de atendimento se dá no setor de neurologia.

Considerando que o foco principal dos encaminhamentos dos profissionais de saúde comumente é voltado para os distúrbios de linguagem, o objetivo deste estudo foi realizar um levantamento das possíveis sequelas fonoaudiológicas dos pacientes internados por AVC nas enfermarias do HPB, destinando-se diretamente à ampliaçáo do conhecimento destes profissionais a respeito de tal tema e auxiliando-os, assim, em futuros encaminhamentos e atendimentos a esta 
população, dada a importância do profissional da fonoaudiologia frente a estas sequelas.

\section{MÉTODO}

\section{Amostra}

Este estudo é observacional, transversal individual, não comparado e contemporâneo. Realizado com 26 pacientes, com idade entre 48 e 77 anos, de ambos os gêneros, com diagnóstico de AVC, internados no HPB, no período de março a maio de 2007. Este projeto foi aprovado pelo Comitê de Ética e Pesquisa do Centro Universitário Metodista - IPA, com número de protocolo 305 em 01/03/2007.

Foram considerados como critérios de inclusão os pacientes com diagnóstico clínico médico de AVC e estarem internados nas unidades de enfermaria do HPB. E entre os critérios de exclusão, considerou-se não ter assinado o TCLE e recebimento de alta hospitalar antes da realização de avaliação fonoaudiológica, pacientes com outros transtornos neurológicos associados, clinicamente não estáveis, necessidade de suporte respiratório (pacientes entubados, traqueostomizados), coma, transtornos psiquiátricos, transtornos congênitos.

As razóes da internaçáo hospitalar dos pacientes desta pesquisa, consistem no controle das condiçóes clínicas e alimentares, realização de medicação, entre outros cuidados médicos e da equipe de saúde.

Os pacientes foram selecionados de forma aleatória, considerando itens de exclusão, para avaliação fonoaudiológica a partir do diagnóstico médico de AVC nas unidades de enfermaria do HPB. Após esta seleção preliminar, os familiares foram informados da possibilidade de participaçáo neste estudo, cientes de todo o processo de avaliação e, dessa forma, aceitaram ou não assinar o Termo de Consentimento Livre e Esclarecido (TCLE). Só através deste termo assinado, o paciente participou da pesquisa.

\section{Procedimento}

O desfecho e seus dados de avaliação fonoaudiológica estáo relacionados a dados estatísticos publicados.

Os dados obtidos na pesquisa foram coletados através do protocolo de Avaliação Fonoaudiológica das Disfagias Orofaríngeas de Etiologia Neurogênica $(A F D N)^{18}$. Este protocolo é utilizado para avaliação fonoaudiológica dos pacientes internados nas enfermarias do HPB que foram acometidos por doenças neurogênicas.

A avaliação fonoaudiológica aconteceu de acordo com o protocolo AFDN. Este foi aplicado pela aluna pesquisadora, ou ainda, foi recolhido junto aos grupos de $6^{\circ}$ e $7^{\circ}$ semestres do curso de Fonoaudiologia do Centro Universitário Metodista - IPA, que realizam estágio curricular supervisionado nas enfermarias do HPB. Neste hospital são atendidos principalmente pacientes do Sistema Único de Saúde (SUS), no qual são internados aqueles acometidos por AVC, Traumatismo Cranioencefálico, Tumor Cerebral e Discopatias. No mesmo local atuam a equipe médica, equipe de enfermagem e os estagiários que compóe os estágios supervisionados do Centro Universitário Metodista IPA, dos cursos de Fonoaudiologia, Fisioterapia, Terapia Ocupacional, Nutrição e Enfermagem.

\section{Material}

$\mathrm{O}$ protocolo AFDN trata-se de uma avaliação clínica fonoaudiológica a ser realizada junto ao leito que busca estabelecer a ocorrência de disfagia, estipulando o seu tipo em oral, faríngea ou orofaríngea e o seu grau de comprometimento em leve, moderada e severa. Este protocolo contempla dados quanto ao nível de elaboração mental do paciente, aspectos da linguagem e das estruturas e funçóes orofaciais. Ele engloba, também, os níveis de saturação periférica de oxigênio $\left(\mathrm{SpO}_{2}\right)$ antes, durante e após a deglutição coletados através de um oxímetro de pulso e aspectos da ausculta cervical no momento da deglutiçáo das diferentes consistências de alimentos.

O critério adotado para inclusão dos pacientes nos grupos acometidos por disfagia e/ou alteraçóes de linguagem foi estabelecido através do protocolo de avaliação AFDN.

A oximetria de pulso é um dos métodos instrumentais complementares e rotineiramente utilizados na 
avaliação das disfagias. Este é utilizado para evidenciar as possíveis alteraçôes de oxigênio-hemoglobina e fornecer informações sobre a saturação de oxigênio, ao mesmo tempo em que se relaciona com a ventilaçáo, desde que o nível de oxigênio inspirado esteja normal. Utiliza-se como inferência dos sinais de aspiração através dos níveis de saturação do sangue arterial. É um método simples, não-invasivo, colocado no dedo do paciente por alguns segundos ${ }^{14,19,20}$.

Considera-se como dessaturação a variação dos índices de saturação $\geq$ a $2 \%$. A alteração $\geq$ a $2 \%$ na saturação de oxigênio durante a deglutição revelava a dificuldade e demonstrava a importante correlação entre dessaturação de oxigênio e aspiração durante os dois minutos após a ingestáo do alimento ${ }^{21,22}$.

A importância e eficiência do uso do oxímetro de pulso como complemento da avaliaçáo das disfagias estấo evidenciadas na relação da dessaturação à disfagia e essa provocar um comprometimento do sistema respiratório em pacientes disfágicos ${ }^{23}$.

O protocolo AFDN é realizado, em média, em 30 minutos, num único momento com o paciente.

\section{Análise Estatística}

A análise dos dados deste trabalho foi realizada através da estatística descritiva (média, mediana, moda, desvio padrão, mínimo, máximo, frequências e percentuais) e verificada através do teste Qui-quadrado. Nas conclusóes destes dados utilizou-se o nível de significância de 5\% ( $\mathrm{p} \leq 0,05)$. Os dados foram computados no programa SPSS, versão 15.0.

\section{RESULTADOS}

Participaram do estudo 26 pacientes com diagnóstico de AVC. A idade média dos pacientes é de 63,03 (dp $\pm 12,91$ anos).

O tempo entre o evento neurológico e a detecção dos distúrbios, através da avaliação fonoaudiológica foi, em média, de 2,7 dias. A extensão da lesão e sua localização não compuseram os objetivos deste estudo, por ter sido este, o de levantamento da divergência na prevalência dos encaminhamentos para o setor de fonoaudiologia.
Os pacientes apresentavam lesóes sugestivas de hemisfério direito e/ou esquerdo, entretanto, não foi computada a extensão do comprometimento neurológico, pois vários resultados de tomografias cerebrais não estavam laudadas, desta forma, apenas foi considerado o diagnóstico estabelecido pelo médico responsável.

Participaram desse estudo 14 pacientes do gênero masculinos (12 brancos e 2 negros) e 12 do gênero feminino (8 brancas e 4 negras) A relação entre tais dados foram considerados estatisticamente não significativos.

Para a variável tipo de AVC foram encontrados $23(88,5 \%)$ pacientes com AVC Isquêmico e 3 com AVC Hemorrágico (11,5\%).

Quando os dados desta variável, ou seja, tipos de AVC, foram relacionados à variável disfagia acompanhada de Variação de $\mathrm{SpO}_{2}$, apresentaram diferença significativa entre as variáveis $(\mathrm{p}=0,22)$.

Ao serem analisadas as variáveis "deglutição normal" e "disfagia", foi verificada presença de deglutiçáo normal em $15(57,7 \%)$ pacientes e de disfagia em 11 pacientes (42,3\%; $\mathrm{p}=0,000$; Tabela 1$)$. A variável "deglutição normal" foi considerada significativa estatisticamente ao ser relacionada com a presença dos distúrbios de linguagem, com presença de disfagia acompanhada de variação de $\mathrm{SpO}_{2}$, e ainda, com disfagia acompanhada de distúrbios de linguagem.

Tabela 1

Número relativo da relação das variáveis deglutição normal e disfagia

\begin{tabular}{cccc}
\hline & $\mathrm{n}$ & $\%$ & $\mathrm{p}$-valor \\
\hline $\begin{array}{c}\text { Deglutiçáo normal } \\
\text { Disfagia }\end{array}$ & 15 & 57,7 & - \\
\hline Total & 11 & 42,3 & - \\
\hline
\end{tabular}

Teste Qui-quadrado com intervalo de confiança de 95\%

$\mathrm{Na}$ análise da variável "deglutição normal acompanhada de distúrbio de linguagem", 24 pacientes $(92,3 \%)$ não tiveram alteraçóes e apenas $2(7,7 \%)$ apresentaram essas características em conjunto $(\mathrm{p}=0,038$; Tabela 2). Quanto a variável "presença de disfagia 
acompanhada de variação de $\mathrm{SpO}_{2}$ ”, 18 pacientes $(69,2 \%)$ apresentaram saturação de oxigênio estável e 8 $(30,8 \%)$ apresentaram variação de saturação maior ou igual a $2 \%(\mathrm{p}=0,246$; Tabela 3$)$.

Tabela 2

Número relativo da relação entre as variáveis deglutição normal e distúrbio de linguagem

\begin{tabular}{cccc}
\hline & $\begin{array}{c}\text { Deglutição normal e } \\
\text { Distúrbios de Linguagem } \\
(\mathrm{n})\end{array}$ & $\%$ & p-valor \\
\hline Apresentou & 2 & 7,7 & - \\
Não apresentou & 24 & 92,3 & - \\
\hline Total & 26 & 100 & 0,038 \\
\hline
\end{tabular}

Teste Qui-quadrado com intervalo de confiança de 95\%

Tabela 3

Número relativo da variável disfagia e variação de saturação periférica de oxigênio

\begin{tabular}{cccc}
\hline & $\begin{array}{c}\text { Disfagia e Variação } \\
\text { de } \mathrm{SpO}_{2} \\
(\mathrm{n})\end{array}$ & $\%$ & p-valor \\
\hline Apresentou & 18 & 69,2 & - \\
Não apresentou & 8 & 30,8 & - \\
\hline Total & 26 & 100 & 0,246 \\
\hline
\end{tabular}

$\mathrm{SpO}_{2}$ - Saturação periférica de oxigênio

Teste Qui-quadrado com intervalo de confiança de 95\%

Do total da população, $6(23,1 \%)$ se enquadraram na variável em que foi relacionada à presença de disfagia acompanhada de distúrbios de linguagem e 20 deles $(76,9 \%)$ não correspondem a estas características $(\mathrm{p}=0,000$; Tabela 4). Foram considerados estatisticamente significativos e estão expostos na Tabela 4.

Quando analisada isoladamente a variação de saturação do total de pacientes, verificou-se incidência de dessaturação em $57,7 \%$ do total de pacientes.

$\mathrm{Na}$ variável "Distúrbios de Linguagem" foram encontrados 8 pacientes $(30,8 \%)$ com presença de distúrbios de linguagem e 18 (69,2\%) sem estes sinais clínicos ( $\mathrm{p}=0,05$; Tabela 5). Ao ser comparada com outras variáveis, verificou-se que a variável "Distúrbio de Lin- guagem" mostra-se estatisticamente significativa frente à variável "disfagia”.

Tabela 4

Número relativo da variável presença de disfagia e distúrbios de linguagem

\begin{tabular}{cccc}
\hline & $\begin{array}{c}\text { Disfagia e Distúrbios } \\
\text { de Linguagem } \\
\text { (n) }\end{array}$ & $\%$ & p-valor \\
\hline Apresentou & 6 & 23,1 & - \\
Não apresentou & 20 & 76,9 & - \\
\hline Total & 26 & 100 & 0,000 \\
\hline
\end{tabular}

Teste Qui-quadrado com intervalo de confiança de 95\%

Tabela 5

Número relativo da variável distúrbio de linguagem

\begin{tabular}{cccc}
\hline & $\begin{array}{c}\text { Distúrbios de } \\
\text { Linguagem } \\
\text { (n) }\end{array}$ & $\%$ & p-valor \\
\hline Apresentou & 8 & 30,8 & - \\
Não apresentou & 18 & 69,2 & - \\
\hline Total & 26 & 100 & 0,05 \\
\hline
\end{tabular}

Teste Qui-quadrado com intervalo de confiança de 95\%

Quanto a sua classificação, dos 8 pacientes $(30,8 \%)$ que apresentaram distúrbios de linguagem, 2 (25\%) tiveram alteração expressiva, 1 (12,5\%) compreensiva e 5 (62,5\%) expressiva acompanhada de compreensiva.

\section{DISCUSSÃO}

A demanda de encaminhamentos ao setor de Fonoaudiologia tem por foco principal o tratamento de distúrbios de linguagem, o que refoge aos objetivos iniciais do setor de fonoaudiologia, voltado para os transtornos de deglutição, devido a seus expressivos riscos.

No mesmo sentido, chamam atenção as diferentes denominaçốes atribuídas pelos profissionais de saúde deste hospital e talvez de outras instituições, aos distúrbios fonoaudiológicos verificados, o que está a demonstrar a relevância dos esclarecimentos aqui expostos. 
Portanto, o objetivo do estudo foi realizar um levantamento das sequelas fonoaudiológicas associadas ao AVC, para que os profissionais da saúde pública tenham conhecimento sobre a demanda que se lhes apresenta e, principalmente, para que os pacientes sejam devidamente encaminhados para tratamento fonoaudiológico.

Nos resultados, tem-se que a idade média dos pacientes avaliados é de 63,03 anos, estes dados têm respaldo na literatura, considerando-se que esta frequência foi encontrada em um estudo com 10.900 pessoas de diversos países sobre Envelhecimento, Saúde e Bem-Estar (SABE) realizado pela Organização PanAmericana da Saúde ${ }^{24}$, que aponta que uma em cada duas pessoas com mais de 60 anos apresentaram fatores de risco para AVC (hipertensão, diabetes, doenças cardíacas) e que mais $80 \%$ dessas pessoas informaram terem tido AVC.

$\mathrm{O}$ número relativo quanto ao gênero dos participantes foi encontrado na literatura com valores similares, de 54\% homens e 46\% mulheres ${ }^{1}$. Em outro estudo, onde foi realizado levantamento de dados de 300 prontuários de pacientes com fator de risco para AVC, 10 apresentaram a doença prévia e 100\% eram homens 5 .

Os transtornos de deglutição são o foco inicial do setor de fonoaudiologia do HPB, estabelecido pelos estágios curriculares em linguagem do adulto - hospitalar, para a triagem fonoaudiológica, pois na literatura tem-se que sequelas nesta função ocorreram em 100\% dos casos com AVC Isquêmico destes, $80 \%$ com deglutição comprometida nas fases oral e faríngea, 16\% somente na oral e $4 \%$ na fase faríngea ${ }^{25}$. Outro estudo $^{26}$ aponta $68,9 \%$ de pacientes com alguma alteração na fase oral e 100\% apresentaram alteraçóes na fase faríngea. Neste grupo foi observada grande frequência de ejeção lentificada ou em dois tempos. Estes dados sugerem importante dificuldade na coordenaçấo entre as duas fases da deglutição, e ainda, um expressivo risco para ocorrência de penetração ou aspiração.

Em estudo recente, a ocorrência do distúrbio de deglutição deu-se em 44,7\% nos $\mathrm{AVC}^{7}{ }^{7}$; outro autor $^{18}$ aponta este fator em $82 \%$ dos casos pesquisados em neuropatias e ainda, foi verificado o distúrbio em
$76,5 \%$ dos pacientes avaliados clinicamente e de $91 \%$ através de avaliação videofluoroscópica ${ }^{6}$. Com a mesma avaliação instrumental, outras pesquisadoras acompanharam 20 sujeitos idosos, pós-AVC, apontando um número bastante elevado (90\%) de disfagia de grau leve a grave. Desse grupo, $40 \%$ demonstraram disfagia grave e expressivos riscos para complicação pulmonar, pela aspiração ${ }^{17}$.

A importância deste foco está além da sua incidência, pois a disfagia pode acarretar alteraçóes nutricionais e comprometimento respiratório, aumentando os índices de mortalidade dos pacientes acometidos por neuropatias ${ }^{14,15,18}$.

A disfagia decorrente do AVC é classificada como neurogênica. Ocorre devido a uma alteração no Sistema Nervoso, responsável pelos processos de coordenaçáo das estruturas envolvidas nas diversas fases da deglutição.

Dos 26 pacientes avaliados no período de dois meses, encontrou-se a presença de disfagia em $42,3 \%$ dos casos, sendo verificado na literatura ${ }^{27}$ que a ocorrência de disfagia é bastante comum após os AVC's, apresentando-se em 51\% dos indivíduos dois dias após o AVC e reduzindo para 27\% após sete dias.

Nesta pesquisa, através do protocolo AFDN, foram realizados levantamentos dos valores de saturação periférica de oxigênio antes e após a avaliação com os alimentos para verificar dessa forma, se ocorreu ou não a variaçáo de $\mathrm{SpO}_{2}$, considerando-se como dessaturação valores em variância a partir de $2 \%$. Do total da população deste estudo, $57,7 \%$ apresentou variação $\mathrm{SpO}_{2}$.

Ao relacionar a $\mathrm{SpO}_{2}$ com os quadros de disfagias e de deglutição normal, verificamos um total de $30,8 \%$ dos pacientes com variação de $\mathrm{SpO}_{2}$. Não foram encontrados dados na literatura com estes referencias.

A literatura mostra que em $45 \%$ (de um total de 40) dos pacientes, acometidos por neuropatias, avaliados em seu estudo apresentaram dessaturação $\geq$ a $2 \%{ }^{18}$.

Embora nos AVC's a afasia seja um sintoma bastante comum, os estudos de Mac-Kay, AssencioFerreira e Ferri-Ferreira (2003) ${ }^{11}$ levam em considera- 
ção na literatura ${ }^{11}$ a classificação e características dos distúrbios de linguagem, não havendo o relato dos prováveis índices estatísticos, o que tornou inviável a correlação destes dados.

A partir desta pesquisa, percebemos que, estatisticamente, os distúrbios de linguagem não são significativos perante as disfagias. Entretanto, a soma dos casos em que os indivíduos apresentam estas duas características relacionadas e comparados à disfagia mostram-se estatisticamente significativos.

Deve-se lembrar que entre os sinais clínicos, decorrentes dos AVC's, são comuns os distúrbios da fala. Estão relacionados aos distúrbios de deglutição ou ainda, ao risco para as disfagias, pois os movimentos motores orais e de fala, da voz e da deglutição, devem constar na história clínica do paciente, e quando alterados, podem estar relacionados à aspiração em adultos com etiologia neurológica, em que os sinais clínicos adicionais aos da história do paciente podem ser descritos como: disartria, tosse após a deglutiçãa, alteraçáo da qualidade vocal, redução do status mental, presença de deglutiçôes múltiplas, dificuldade em gerenciar a ingestâo do bolo alimentar, redução da elevação da laringe e ainda, presença de pneumonia prévia ${ }^{28}$.

Embora considerado, este dado mostra-se relevante para o acompanhamento do paciente junto ao leito. Aqueles que por ventura apresentam tal possibilidade estão relacionados no grupo de deglutição normal.

Entre as sequelas pós-AVC têm-se: os distúrbios motores, os distúrbios de fala ou de linguagem e os distúrbios de deglutição ${ }^{6}$.

Dentre os entraves dignos de relevância, deve ser mencionada a dificuldade em realizar um estudo com enfoque de pesquisa em curto espaço de tempo, o que possa ter interferido no tamanho da amostra. Outro dado é a dificuldade em encontrar o responsável pelo paciente, o que remete a refletir sobre a possibilidade de um problema social no qual os pacientes permanecem internados sem assistência familiar.

Acredita-se que este estudo poderá chamar a atenção das equipes multidisciplinares, em especial às do $\mathrm{HPB}$, porquanto, demonstra a importância da observação de possíveis sinais de sequelas fonoaudiológicas, auxiliando sobremaneira o pronto encaminhamento dos pacientes para avaliação fonoaudiológica. É através de um atendimento precoce e focado nas principais necessidades do paciente que ocorrerá a facilitação dos mecanismos de plasticidade neuronal, implicando em um eficaz processo de reabilitação das sequelas deixadas pelo AVC.

\section{CONCLUSÕES}

Este estudo mostra a possibilidade de maior conhecimento sobre conceito, variações e dependências entre os sinais clínicos das sequelas fonoaudiológicas.

Aponta ainda, que apesar de na prática clínica o principal foco de encaminhamentos para a fonoaudiologia ser para os distúrbios de linguagem, os resultados afirmam que a sequela prevalente no grupo estudado é a disfagia. Este dado chama a atenção para a falta de conhecimento dos outros profissionais envolvidos sobre a atuação fonoaudiológica com os pacientes de AVC.

É fundamental ainda ressaltar que, apesar da importância dos dados estatísticos levantados neste estudo, sua população (26 pacientes) é reduzida. Portanto, acredita-se que seria de grande valia para a Fonoaudiologia, como ciência, se houvessem outros estudos nessa área e com uma população ou amostra mais vasta para que os resultados fossem mais fidedignos.

\section{REFERÊNCIAS}

1.Radanovic M. Características do Atendimento de Pacientes com Acidente Vascular Cerebral em Hospital Secundário. Arq. Neuropsiquiatria 2000;58:99-106.

http://dx.doi.org/10.1590/S0004-282X2000000100015

2.Damiani IT, Yokoo I. Acidente Vascular Cerebral (Endereço na Internet). Saúde em Movimento. (atualizado em: 04/2010; citado em: 04/2010). Disponível em: http://www.saudeemmovimento.com.br/conteudos/conteudo_print.asp? cod_noticia $=44$

3.André C. Manual de AVC. Rio de Janeiro: Revinter, 1999, 232p.

4.Neuwald RJ, Ferri A, Magalhães AV, Zeni KMM. Aspectos gerais no atendimento emergencial dos acidentes vasculares encefálicos isquêmicos. Rev Cient AMECS 1999;8:43-7.

5.Castro JAB de, Epstein MG, Sabino GBS, Nogueira GLO, Blankenburg 
C, Staszko KF, et al. Estudo dos principais fatores de risco para acidente vascular encefálico. Rev Bras Clin Med 2009;7:171-3.

6.Schelp AO, Cola PC, Gatto AR, Silva RG da, Carvalho LR de. Incidência de Disfagia Orofaríngea Após Acidente Vascular Encefálico em Hospital Público de Referência. Arq. Neuropsiquiatr 2004;62:503-6.

http://dx.doi.org/10.1590/S0004-282X2004000300023

7.Xerez DR, Carvalho YSV, Costa MMB. Estudo Clínico e Videofluoroscópico da Disfagia na Fase Subaguda do Acidente Vascular Encefálico. Radiol. Brás 2004;37:9-14.

8.Lamônica DAC, Minervino-Pereira ACM, Ferreira GC. Conversando sobre afasia: guia familiar. Bauru: Edusc, 2000, 37p.

9.Block F, Kastrau F. Primary progressive aphasia. Nervenarzt 2004;75:1 167-71. http://dx.doi.org/10.1007/s00115-004-1770-z

10.Maciel JAJ. Processamento da linguagem: modelos anátomo-funcionais. In: Nitrini R, Caramelli P, Mansur L. Neuropsicologia: das bases anatômicas à reabilitação. São Paulo: FMUSP, 2003, p.171-81.

11.Mac-Kay APMG, Assencio-Ferreira VJ, Ferri-Ferreira TMS. Afasias e Demências: Avaliação e Tratamento Fonoaudiológico. São Paulo: Ed. Santos, 2003, 102p.

12.Ortiz KZ.(Org.). Distúrbios Neurológicos Adquiridos: Fala e Deglutiçấo. Barueri: Ed. Manole, 2006, 303p.

13. Macedo Filho ED de, Gomes GF, Furkim AM. Manual de cuidados do paciente com disfagia. São Paulo: Lovise, 2000, 127p.

14.Moschetti MB. Disfagia Pós-Acidente Vascular Cerebral - Estudo de um caso. São Paulo: Rev CEFAC 2001;3:133-41.

15.Furkim AM, Mattana A. Disfagia Orofaríngea Neurogênica. São Paulo: Revista da Fonoaudiologia, 2004;56:21.

16. Teixeira INDO. O envelhecimento cortical e a reorganização neural após o acidente vascular encefálico (AVE): implicações para a reabilitação. Ciência \& Saúde Coletiva 2008;13(Sup 2):2171-8.

17.Gatto AR, Rehder MIBC. Comparação entre queixas de deglutição e achados videofluoroscópicos no paciente pós Acidente Vascular Encefálico. Rev CEFAC 2006;8:320-7.

18.Cardoso MCAF. Disfagia Orofaríngea de Causa Neurogênica: Verificação do Índice de Saturação de O2 na Avaliação Clínica Fonoaudiológica
[Dissertação de Mestrado]. Santa Maria: UFSM, 2004, 167p.

19.Kirby RR, Taylor RW, Civetta JM. Manual de Terapia Intensiva. 2a. ed. São Paulo: Manole, 2000, 797p.

20.Pryor JA, Weber BA. Fisioterapia para problemas respiratórios e cardíacos. 2a. ed. Rio de Janeiro: Guanabara-Koogan, 2002, 384p.

21.Collins MJ, Bakheit AM. Does pulse oximetry reliably detect aspiration in dysphagic stroke patients? Stroke, 1997;28:1773-5.

22.Lim SHB, Lieu PK, Phua SY, Seshadri R, Venketasubramanian N, Lee $\mathrm{SH}$, et al. Accuracy of bedside clinical methods compared with Fiberoptic endoscopic examination of swallowing (FEES) in determining the risk of aspiration in acute stroke patients. Dysphagia 2001;16:1-6.

http://dx.doi.org/10.1007/s004550000038

23.Colodny N. Comparation of dysphagics and nondysphagics on pulse oximetry during oral feeding. Dysphagia 2000;15:68-73.

24. World Health Organization. Health and aging (with reference to Resolutions CE130/15 Port., of the 130 Session of the Executive Committee). (Endereço na Internet). Washington, DC: Pan American Health Organization - PAHO (atualizado em: 2010; citado em: 2010). Disponível em: http://www.paho.org/portuguese/gov/csp/csp26-13-p.pdf).

25.Silva RG, Vieira MM. Disfagia orofaríngea neurogênica em adultos pósacidente vascular encefálico: identificação e classificação. In: Macedo Filho E, Pisani JC, Carneiro J, Gomes G. Disfagia: abordagem multidisciplinar. 3a ed. São Paulo: Frôntis Editorial, 1999, p.29-46.

26.Yamada EK, Siqueira KO de, Xerez D, Koch HA, Costa MMB. A influência das fases oral e faríngea da dinâmica da deglutiçăo. Arq Gastroenterol 2004;41:18-23.

http://dx.doi.org/10.1590/S0004-28032004000100004

27.Smithard DG, O'Neill PA, England RE, Park CL, Wyatt R, Mantin DF, et al. The natural history of dysphagia following a stroke. Dysphagia 1997;12:188-93.

http://dx.doi.org/10.1007/PL00009535

28.McCullough GH, Wertz RT. Sensivity and specificity of clinical/bedside examination signs for detecting aspiration in adults subsequent to stroke. Journal of Communication Disorders 2001;34:55-72.

http://dx.doi.org/10.1016/S0021-9924(00)00041-1 